\title{
REVERBERATION MORPHING USING CEPSTRUM DECOMPOSITION Y. Takahashi ${ }^{1}$, M. Tohyama ${ }^{2}$, and H.Nomura ${ }^{3}$ \\ ${ }^{I}$ Waseda University, Tokyo, Japan \\ ${ }^{2}$ Waseda University, Tokyo, Japan, University of York, York, UK \\ ${ }^{3}$ Kure National College of Technology, Hiroshima, Japan
}

\begin{abstract}
Reverberation morphing from an impulse response is an inevitable 3D-audio technology as well as reverberation rendering for an immersive communication network. This paper proposes a method for reverberation morphing by relocating the poles and zeros of the transfer function. It assumes that the distance of the pole/zero locations from the unit circle in the z-plane represents the reverberation condition for the transfer function. Exponential timewindowing moves the minimum-phase zeros, while the pole/zero relocation for the all-pass part is possible by the windowing after causal and non-causal cepstrum decomposition. We confirmed that the reverberation effects on the frequency response rendered by the proposing method were similar to those for recorded impulse responses in a variable reverberation room. This morphing is also applied to stable inverse filtering in a reverberant space.
\end{abstract}

\section{INTRODUCTION}

An immersive communication system requires rendering of auditory events including 3-D spatial sound to enable effective collaboration through an interactive sound field network [1-3]. Rendering and morphing of room reverberation are crucial technology of auditory events modeling in virtual acoustics. This paper describes a 3D-sound morphing by reverberation control using cepstrum decomposition.

Reverberators have been investigated as an effective tool for sound recording and reproducing since many years ago[4]. However reverberators that naturally sound such as real-room reverberation are still under study. Tohyama et al.[5] proposed a new method for generating an impulse response which represents reverberation characteristics based on superposition of free-oscillations whose frequencies are determined based on the modal statisitics.

In practical applications for room acoustics simulation or virtual acoustics, it is required to change the simulation parameters, or to modify the impulse response record in a real room. The proposed method by Tohyama et al.[5] includes various acoustic parameters, however, it is not easy to change the acoustic conditions after generating the impulse response.

For that purpose this paper describes a method for reverberation control according to relocating the poles and zeros of the transfer function. Tohyama et al.[6] investigated the density of the non-minimum phase zeros in the complex frequency plane using a modal expansion form of the room transfer function. However, movement of the zeros due to the reverberation is still under study.

We will introduce a different model from the previous one[6] for the movement of poles and zeros. According to this model, it is possible to modify the impulse response records using cepstrum decomposition and exponential time-windowing. The modification of impulse response can be also applied to realize stable inverse filtering for sound transmission equalization.

\section{TRANSFER FUNCTION IN A ROOM}

The transfer function in a room with small damping (short reverberation) can be represented using the modalexpasion form such as[6]

$$
H(\omega)=\operatorname{const} \sum_{m} \frac{\varphi_{m}\left(\mathbf{x}_{\mathrm{s}}\right) \varphi_{m}\left(\mathbf{x}_{\mathrm{o}}\right)}{\omega^{2}-\omega_{m}^{2}+j \omega \omega_{m} \eta_{m}}
$$

where $\varphi_{m}$ is mode shape, $\omega_{m}$ is the undamped resonance (angular) frequency, and $\eta_{m}$ is the loss factor. The poles of this function are at $\omega= \pm \omega_{m}+j(1 / 2) \omega_{m} \eta_{m}$. If we assume that the reverberation time $T_{R}=6.91\left(2 / \omega_{m} \eta_{m}\right)$ is the same for all modes, then the poles lie along the straight line at a distance $\delta_{0}=6.91 / T_{R}$ above the real frequency axis as shown in Fig.1. If the reverberation time depends on frequency, then the pole line will have a different locus.

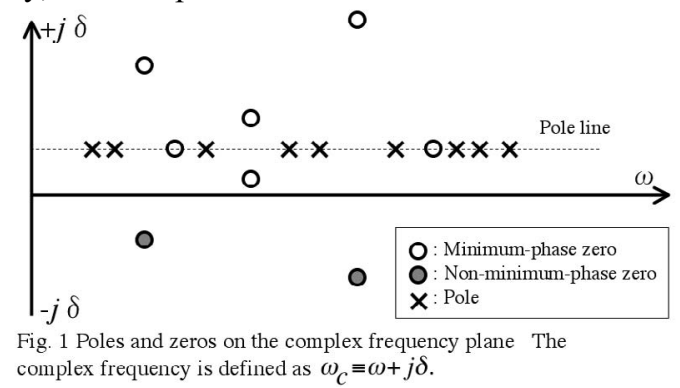

The poles are located on the pole line, but the zeros can be distributed in the complex frequency plane. If the mode shape functions are real, and the pole is as shown in Fig.1, then by symmetry the zeros are evenly distributed above and below the pole line. The density of the nonminimum phase zeros in the complex frequency plane decreases inversely proportional to square of the distance from the pole-line, and thus the accumulated phase trend decrease as the damping increases[6].

The Eq.(1), however, might not be suitable for describing the room TF under wide range of reverberation 
conditions. Since the arrangement of the poles and zeros is based on the "static model", the effect of reverberation is seen only on the distance of the pole-line from the frequency axis, and the symmetry of the pole/zero locations holds. Therefore if we take a short reverberation time (long pole-line distance), then deep dips can be seen in the magnitude response due to isolated non-minimum phase zeros since its density is low.

Consequently the quality of reverberation sound can be greatly changed by the reverberation condition. Magnitude response of the impulse responses is an important signature of the reveberation response different from all-pass reverberators[3][4]. Therefore we will investigate a method for morphing of the impulse response without creating deep dips in the magnitude response including the all-pass phase.

\section{Modification of Magnitude and Phase Responses of the Transfer Function}

3.1Magnitude and Phase of the Transfer Function

Figure 2(a) is a sample of impulse response in a reverberant space and its magnitude 2(b) and phase response 2(c). Although the impulse response has a finite record length, we can see many peaks and dips in the magnitude response and phase changes.

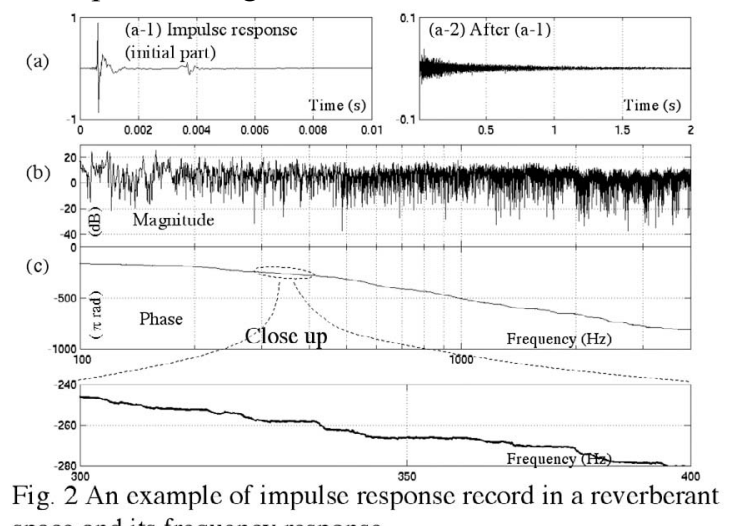

space and its frequency response

We can normally expect that steep(mild) magnitude peaks become mild(steep), as the damping increases(decreases). On the contrary, the dips depend on the location of the zeros, which cannot be estimated at present in a practical situation, and thus the phase trend is not predictable as the reverberation condition varies. It might be possible only according to the "static model" proposed by Tohyama et al.[6]. Therefore we newly propose a method for controlling the peaks and dips in the magnitude response as well as the phase drift from the phase trend.

We assume the perception of reverberant phase depends on the group delay rather than the global phase trend. In our proposed method, therefore, the phase trend remains unchanged even after the modification. The method is based on a view of the entire map for the poles and zeros in the $z$-plane such that they are separate from (close to) the unit circle (instead of the pole-line) without changing their distributions, when the damping becomes large(small).

\subsection{Modification of the Magnitude Response}

The transfer function can be decomposed into the minimum-phase and all-pass components. Figure 3 is a sample of impulse response (Fig.3 (a)) with the decomposition into the minimum-phase (3(b)) and all-pass (3(c)) components in terms of poles and zeros. Relocating the poles and zeros as illustrated in Figs 3(b) and 3(c), the magnitude and phase can be controlled.
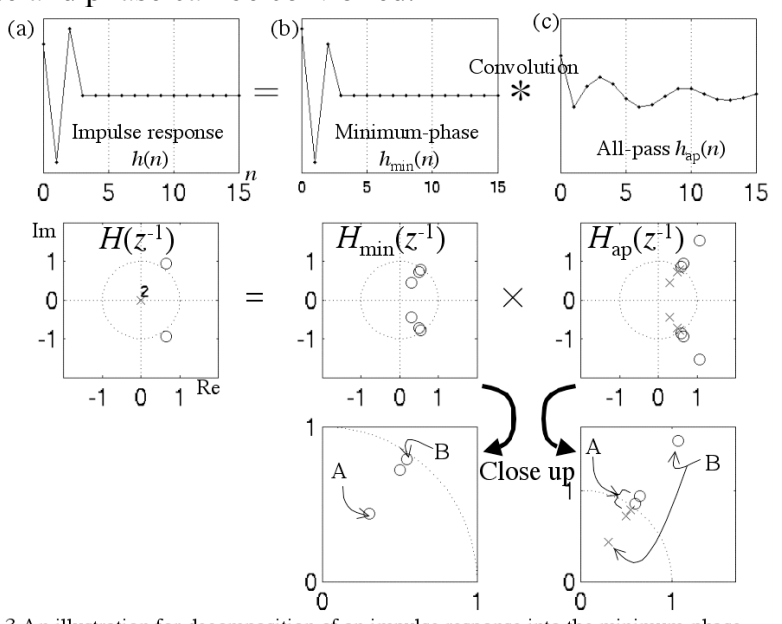

Fig. 3 An illustration for decomposition of an impulse response into the minimum-phase and all-pass components. Top: Time response, Bottom: Poles and zeros on the $z$-plane Poles and zeros are relocated for morphing. $O$ : Minimum-phase zero $\quad x$ : Pole

Exponential time-windowing of the minimum-phase component performs the magnitude modification. Figure 4 shows the magnitude response of with and without the windowing, The windowing with a negative(positive) exponent moves the zeros farther from(close to) the unit circle. Plot $\mathrm{A}$ is modified with the negative exponent, and $\mathrm{B}$ is obtained with the positive one.

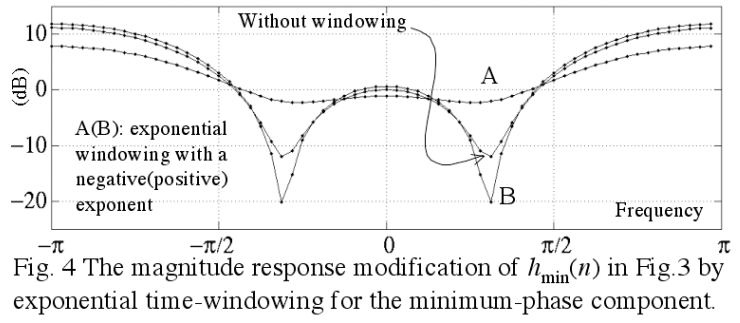

\subsection{Modification of the All-Pass Phase}

Not only the minimum-phase (magnitude) response but the all-pass phase is important for reverberation sound rendering. Relocating the poles and zeros of the all-pass response such as shown in Fig.3(c) can be done using the cepstrum.

Figures 5(a) and (b) show the all-pass impulse response shown in 3(c) and its cepstrum, respectively. Figure 5(c) is a newly extracted time-response from the causal part of the cepstrum shown in Fig.5(b). This time-response could be interpreted as the impulse response due to the poles of 
the all-pass. Figures 5(d) and (e) are the exponentiallywindowed all-pass-pole impulse response and its cepstrum, respectively. We can get the phase cepstrum as shown in Fig.5(f) by adding its non-causal part into the causal cepstrum by Fig.5(e). The impulse response can be constructed from the all-pass phase cepstrum as shown in Fig.5(g). Figure 6 illustrates the all-pass phase modification corresponding to the minimum-phase magnitude shown in Fig.4. The abrupt phase changes are not seen in plot A.

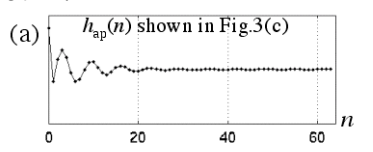

(b)
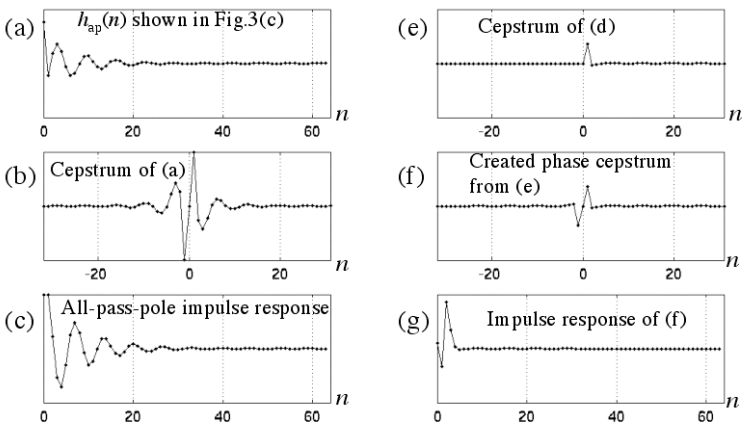

(d) Exponential windowing of (c)

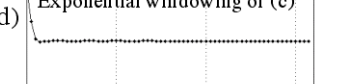

Fig. 5 All-pass phase modification: pole/zero relocating using the cepstrum

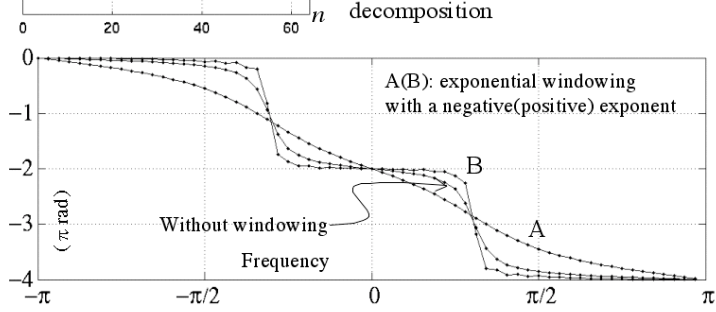

Fig. 6 All-pass phase modification corresponding to the magnitude in Fig. 4

\section{MORPHING OF ROOM REVERBERATION}

Let' $s$ take the example shown in Fig.2. Figure 7 presents the minimum-phase magnitude and all-pass phase responses after the morphing by exponential timewindowing with a negative exponent. We can confirm similar effect to Figs. 4 and 6 on the minimum-phase and all-pass components.

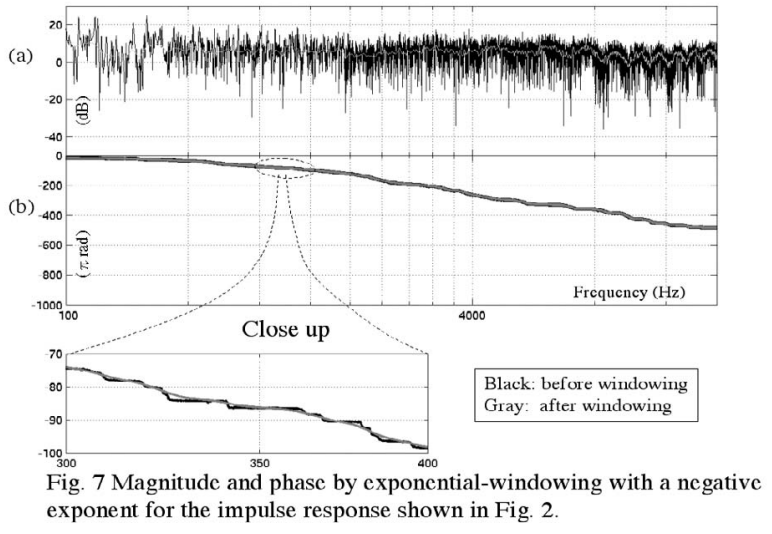

The modification procedure is summarized in Fig.8. When we add reverberation effect on the minimum-phase response using the windowing with a positive exponent, again we have to decompose the windowed response into the minimum-phase and all-pass components as shown in Fig.8. This is because the windowed minimum-phase with a positive exponent can be of non-minimum phase. The newly created all-pass response is convolved with the original all-pass part.

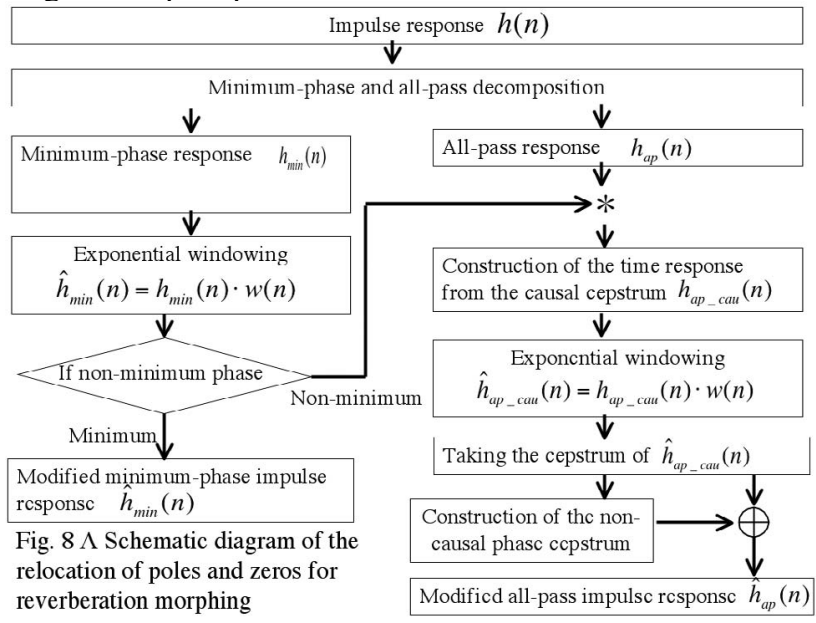

Figure 9 shows examples of impulse responses and their frequency responses for a reverberation variable room under live and dead (noted by OL and OD) conditions. The response denoted by ML(MD) represents the morphing result by adding (decreasing) the reverberation into OD (from OL). Although the details in the responses are different between the original and morphing records, we can find the similar reverberation effects between them. The frequency response rendered by morphing is smoother than the original one when the reverberation is decreased. It might be desirable for virtual acoustics control.
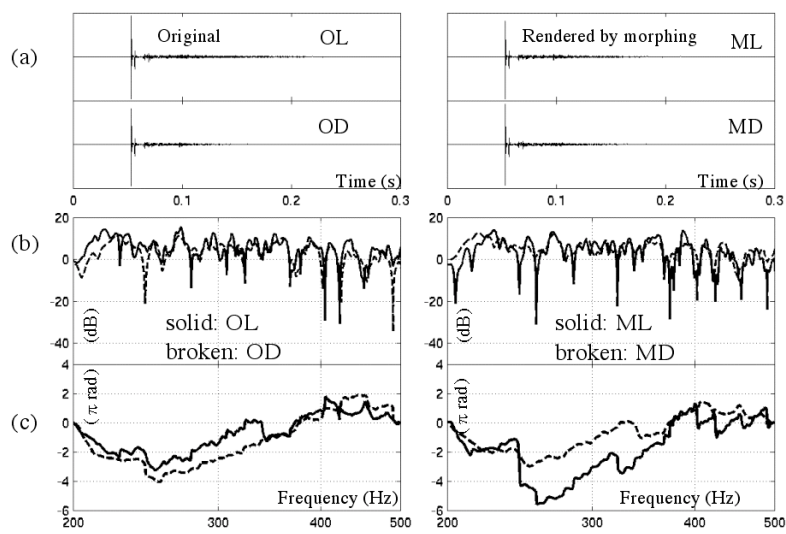

Fig. 9 Morphing examples of reverberation for live (OL) and dead (OD) rooms according to the procedure shown in Fig. 8

ML: Reverberation increasing into OD, MD: Decreasing reverberation from $\mathrm{OL}$ (a) Impulse response (b) Mnimum-phase magnitude (c) All-pass phase

\section{STABLE INVERSE FILTERING IN A REVERBERANT SPACE}

Morphing of the transfer function described above can be used for stable inverse filtering. Inverse filtering can be instable in a reverberant space[7][8]. Figure 10 shows a schematic of sound reproduction and equalization by in- 
verse filtering in a reverberant space. Suppose that we have the inverse filter for the target position. Figures 11(a)-(d) illustrate samples of input and reverberant speech signals at the target and other positions in a local area, and similarly Figs.11(e)-(g) shows the receiving signals reproduced after the inverse filtering. We can see that inverse filtering is possible at the target with a time delay(not included in the Figure); however, reproduced signals might be deteriorated at other positions even in a local area by the target-oriented inverse filtering.

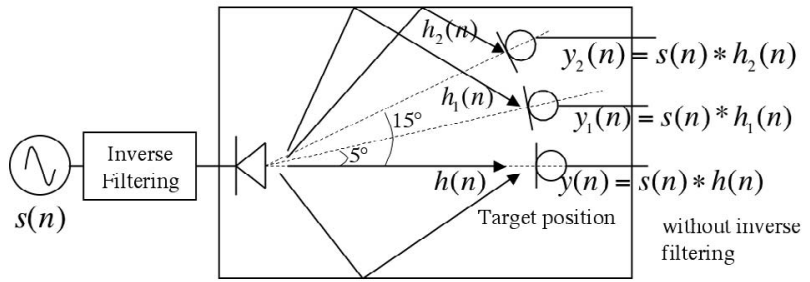

Fig. 10 A schematic of the inverse filtering for sound reproduction and equalization

Figures 12(a)-(c) present the inverse filtering results after relocating the poles and zeros of the transfer function at the target position following the procedure shown in Fig.8. We can expect the equalization at the target might be possible, which is not harmful at other positions.

\section{SUMMARY}

We have described a method for reverberation morphing based on relocating the poles and zeros of the transfer function. Different from the modal theory, we assumed that the distance of the pole/zero locations from the unit circle in the $z$-plane represented the reverberation condition for the transfer function. The proposed method can be performed separately; by exponential time-windowing for the minimum-phase part, and the same windowing for the non-minimum components after causal and non-causal cepstrum decomposition. We confirmed that the reverberation effects on the frequency response rendered by the proposing method were similar to those for recorded impulse responses in a variable reverberation room. This morphing can be also applied to stable inverse filtering in a reverberant space, and also expected to be an experimental tool for auditory research on the 3D spatial sound. The authors would thank NHK Science \& Technical Research Laboratories for permission of the impulse response records in the reverberant variable room.

\section{REFERENCES}

[1] L. Sacioja et al., "Creating Interactive Virtual Acoustic Environments”, J. Audio Eng. Soc., vol. 47 pp. 675-705 (1999)

[2]C. Kyriakakis et al., "Surrounded by Sound", IEEE Signal Processing Magazine, vol. 1, pp. 55-66 (1999)

[3] Y. Takahashi, M. Tohyama. M. Matsumoto, and H. Yanagawa, "An Auditory Events Modeling Language(AEML) for Interactive Sound Field Network," ICA 2003, Tu.5 D.5

[4]M. R. Schroeder, "Improved Quasi-Stetreophony and "Colorless" Artificial Reverberation." J. Acoust Soc. Am, vol. 33 pp.1061-1064 (1961)

[5] M. Toyama, M. Kazama, and Y. Kamiya, “3-D Reverberation Sound Rendering Based on Distribution Statistics of Poles and Residues in Transfer Functions," 17th Int. Congress on Acoustics 4B.11.04

[6] M. Tohyama, R. H. Lyon and T. Koike, "Reverberant Phase in a Room and Zeros in the Complex Frequency Plane”, J. Acoust. Soc. Am. 89, pp.1701-1707, 1991

[7] O. Kirkeby and P. A. Nelson, "Digital Filter Design for Inversion Problems in Sound Reproduction," J. Audio Eng. Soc. 47. pp.583-595 (1999)

[8] P. D. Hatziantoniou and J. N. Mourjopoulos, "Errors in Real-Time Room Acoustics Dereverberation," J. Audio Eng. Soc. 52(9) pp. 883-899 (2004)

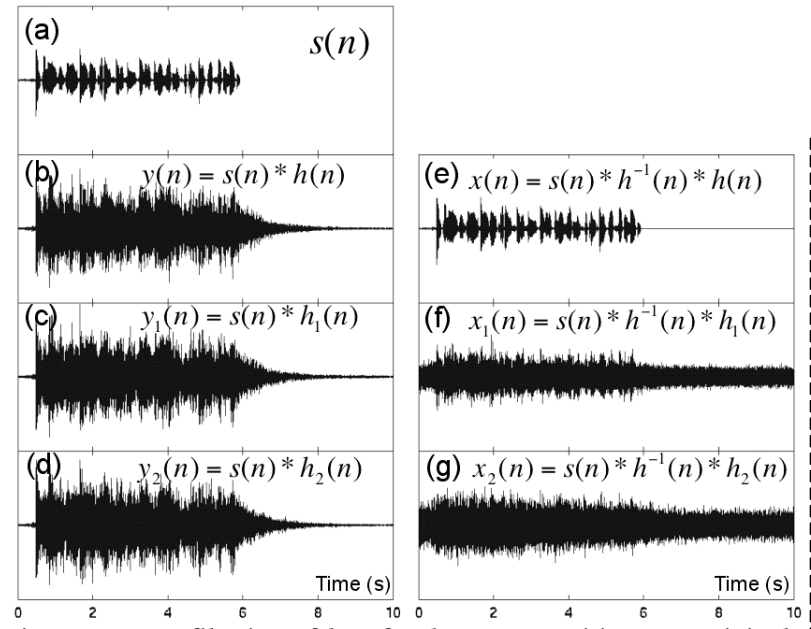

Fig. 11 Inverse filtering of $h(n)$ for the target position. (a) Original dry speech (b-d) reverberant speech without the inverse filter (e-g) reverberant speech with the inverse filter.

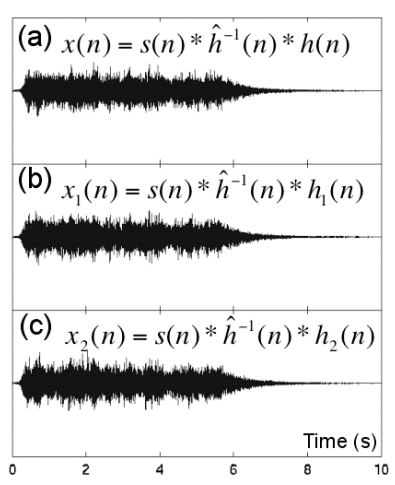

Fig. 12 Inverse filtering for the impulse response $\hat{h}(n)$ modified following the procedure in Fig.8. 